\title{
The global preclinical antibacterial pipeline
}

Ursula Theuretzbacher $\mathbb{1}^{1 *}{ }^{*}$, Kevin Outterson $\mathbb{D}^{2,3}$, Aleks Engel $\mathbb{1}^{4}$ and Anders Karlén $\mathbb{1}^{5}$

Abstract | Antibacterial resistance is a great concern and requires global action. A critical question is whether enough new antibacterial drugs are being discovered and developed. A review of the clinical antibacterial drug pipeline was recently published, but comprehensive information about the global preclinical pipeline is unavailable. This Review focuses on discovery and preclinical development projects and has found, as of 1 May 2019, 407 antibacterial projects from 314 institutions. The focus is on Gram-negative pathogens, particularly bacteria on the WHO priority bacteria list. The preclinical pipeline is characterized by high levels of diversity and interesting scientific concepts, with 135 projects on direct-acting small molecules that represent new classes, new targets or new mechanisms of action. There is also a strong trend towards non-traditional approaches, including diverse antivirulence approaches, microbiome-modifying strategies, and engineered phages and probiotics. The high number of pathogen-specific and adjunctive approaches is unprecedented in antibiotic history. Translational hurdles are not adequately addressed yet, especially development pathways to show clinical impact of nontraditional approaches. The innovative potential of the preclinical pipeline compared with the clinical pipeline is encouraging but fragile. Much more work, focus and funding are needed for the novel approaches to result in effective antibacterial therapies to sustainably combat antibacterial resistance.

Lead generation phase (hit-to-lead phase) Drug discovery phase where promising molecules (hits) are evaluated and undergo limited optimization to identify suitable lead compounds.
${ }^{1}$ Center for Anti-Infective Agents, Vienna, Austria.

${ }^{2}$ Boston University, Boston, MA, USA.

${ }^{3}$ CARB-X, Boston, MA, USA.

${ }^{4}$ Novo Holding $A / S$,

Copenhagen, Denmark.

${ }^{5}$ Department of Medicinal Chemistry, Uppsala University, Uppsala, Sweden.

*e-mail: utheuretzbacher@

cefaia.com

https://doi.org/10.1038/

s41579-019-0288-0
Resistance to antibiotics is a natural phenomenon that has been noted since the introduction of penicillin in the $1940 \mathrm{~s}^{1}$. Whenever clinically relevant resistance has emerged, the problem has been tackled with modification of existing antibiotic classes with limited crossresistance to existing drugs or introduction of new classes $^{2}$. The relative ease of the early antibiotic discovery programmes and the financial rewards that followed created a wasteful and uncritical use of antibiotics without adequate consideration of the societal consequences ${ }^{3}$. After this 'golden antibiotic era', large pharmaceutical companies faced major scientific challenges searching for new antibiotics, especially with regard to penetration barriers and efflux mechanisms in Gram-negative bacteria requiring high antibiotic doses with potential associated toxicity issues. These companies finally abandoned antibacterial drug discovery activities beginning in the 1980s. Furthermore, they lost interest in a field that did not promise ever-increasing market growth and profits. Exits by large pharmaceutical companies have caused concern among scientists, the health-care community, civil society advocates and policymakers ${ }^{5,6}$. Because of the long timelines for research and development, urgently needed responses and action can be calibrated only by knowing the global activities (and lack of activities) in antibacterial drug development. This mapping activity was initiated by the WHO with a recently published global clinical antibacterial pipeline report? In contrast to the clinical pipeline, less is known about the preclinical antibacterial pipeline. In this Review, we analyse the preclinical antibacterial pipeline and provide a current snapshot and decision support for all actors in this field and some information on the broader context.

To assess the global preclinical bacterial pipeline, we considered all projects from several databases and research and development programmes (BOX 1) and included all antibacterial projects that were at least in the lead generation phase (hit-to-lead phase) but had not yet reached first-in-human studies. We grouped all preclinical projects that met these criteria into the following categories: direct-acting traditional agents (traditional antibiotics that directly inhibit growth or kill the bacteria); antibacterial vaccines, antibodies and antibody-drug conjugates; phages or phage-derived proteins and microbiota-modulating therapies; antivirulence agents that augment other agents; potentiators that enhance and augment or transform other agents; repurposed approved drugs; and immunomodulators that are developed for a bacterial disease $e^{8-15}$. We also looked at the type and location of institutions carrying 


\section{Box 1 Assessment criteria of the global preclinical antibacterial pipeline}

The basis of this Review was five databases or programmes with information about antibacterial preclinical research and development projects: the Center for AntiInfective Agents (CEFAIA; 1235 data), CARB-X funding proposals (804), REPAIR Impact Fund funding proposals (80), ENABLE (10 projects with data provided by project owners) and the Joint Programming Initiative on Antimicrobial Resistance (JPIAMR; 20 projects). No projects from JPIAMR could be included because they did not meet the inclusion criteria. The data span from September 2016 to February 2019 and all data were updated if possible, with a cut-off date of 1 May 2019. In the case of overlapping data, the most recent update was used. The sources for updates were confidential personal communication, scientific abstracts, company websites, press releases and scientific and commercial publications. Whereas the JPIAMR, ENABLE and REPAIR Impact Fund data were restricted geographically, the data from CEFAIA and CARB-X were global in scope. Institutions were categorized as small and medium-sized enterprises (fewer than 1,000 employees), large companies (more than 1,000 employees), academic and other publicly funded institutes, supported by philanthropic organizations or non-profit institutions, and public-private partnerships. Companies and universities were counted as a single institution even if they have subsidiaries in different countries or different departments within a university. Israel was included in Europe for categorization purposes owing to the strong research ties. The inclusion criteria require the project to target bacterial infections and to be in the discovery (hit-to-lead and lead optimization phases ending with declaring a preclinical candidate) and preclinical development phases for submission of an application for clinical trial authorization (CTA) or an investigational new drug application (IND), often called 'CTA/IND-enabling studies'. Duplicates of programmes due to collaborations, acquisitions or licensing were eliminated. Projects were included only if the product had not had a first dose in humans before 1 May 2019 as evidenced by the aforementioned sources for updates and public clinical trial registries. Also excluded were antibacterial products for non-human uses, diagnostics, medical devices, conventional vaccines not focused on resistant pathogens (such as vaccines against pneumococci, meningococci, Haemophilus influenzae), new formulations and delivery methods of approved drugs (unless they allow new antibacterial use that was not possible before), projects for label expansion of a product already marketed or in clinical development, immunomodulators if not developed for a specific bacterial disease, wound care products unless used as a first model to assess the potential for other clinical indications, disinfectants and antibacterial ions. All data on institutions and their programmes were anonymized and aggregated to prevent tracking of data to specific companies or projects, as some companies request confidentiality during preclinical stages.

\section{Repurposed approved drugs \\ Repurposing a drug is a strategy for identifying new uses for an approved drug that are outside the scope of the original indication. \\ Label expansion \\ Aims to achieve additional regulatory approval for a new indication beyond the original use for which the drug was approved.}

\section{Indications}

A therapeutic indication refers to the use of a drug for treating a particular disease. The indication can be approved by regulatory agencies or not approved.

Spectrum

Range of activity against a group of bacteria. out the project. Finally, we further assessed the planned indications, spectrum and formulations and the stage of the project.

Overall, the current preclinical antibacterial pipeline consists of 407 highly diverse projects from 314 institutions, most of which are small and medium-sized enterprises (SMEs). Less than half of the projects involve direct-acting small molecules and encouragingly $70 \%$ of these aim at new targets (FIG. 1). In the following sections, we discuss the characteristics of the institutions and then go through the different project classes, highlighting the risks and potential of the preclinical antibacterial pipeline.

\section{Institutions}

Three hundred and fourteen research and development institutions are working on at least one preclinical antibacterial programme that met our inclusion criteria (BOX 1; FIC. 2a). Most of these institutions are SMEs, comprising 255 companies ( $81 \%$ of all institutions), and most of these SMEs are based in North America (United States and Canada; 56\%) and Europe (including Israel; 36\%). European SMEs were found most often in the United Kingdom, followed by France, Switzerland, Denmark and the Netherlands (FIG. 2b). Although we could not verify the exact number of employees at SMEs in 5\% of cases, at least $60 \%$ of all included SMEs are very small companies with fewer than ten employees. Ninety per cent of the SMEs with a known number of employees $(n=243)$ are small companies with fewer than 50 employees. Only $5 \%$ of the SMEs have more than 100 employees but fewer than 500 employees. These numbers show that the great majority of the world's preclinical antibacterial pipeline is in the hands of very small companies with very limited financial (and workforce) resources.

Given the small size of most SMEs it is not surprising that they predominately focus on only antibacterial research and development, mostly based on one specific technology (Supplementary Fig. 1). A few have additional discovery projects in other anti-infective areas (for example, antivirals). Some SMEs work in one or more additional therapeutic areas, especially immunooncology and/or inflammation. The distribution of these three categories (only antibacterial therapy, only the anti-infective field, or both antibacterial and other therapeutic areas) is similarly distributed among European and North American SMEs.

Other types of institutions besides SMEs included 37 academic institutions, 10 large companies (more than 1,000 employees), 8 non-profit research institutions and 4 public-private partnerships (FIG. 2a). Most academic institutions were excluded as their projects were not advanced enough to meet the inclusion criteria. Very few global pharmaceutical corporations have active clinical development programmes according to their published pipelines (for example, Pfizer, GlaxoSmithKline, Medimmune/AstraZeneca, Genentech/Roche). Most of these companies are not active in preclinical antibacterial research and development, although it is possible that the companies are especially adept at keeping their programmes confidential and did not apply for funding. The large pharmaceutical companies (more than 1,000 employees) included in this study and engaging in preclinical antibacterial research and development are mainly located in Asia and Europe and have a regional focus. From our review of the data, these particular preclinical projects do not represent a renaissance in interest by large companies in antibiotic resistance. Therefore, SMEs carry out the great majority of the pipeline, with few employees and dependence on one programme or technology. This vulnerability is commonly characterized not only by a narrow set of expertise and dependence on the success of a single or a few similar prioritized projects, but also by the need for continued flow of funding, mostly grants, as private funding is relatively modest in preclinical antibacterial research and development. This situation causes high volatility of the number of SMEs and threatens the stability of the early pipeline.

\section{Antibacterial preclinical programmes}

Of the 407 preclinical projects that we identified, 81\% are in SMEs and $4 \%$ are in larger companies, and they fall into seven broad categories (FIC. 3). One hundred and eighty-seven projects (46\%) involve agents that inhibit or kill bacteria directly ('traditional antibiotics'), 33 projects involve phages or phage-derived peptides that affect bacteria directly, 33 projects involve agents that do not 


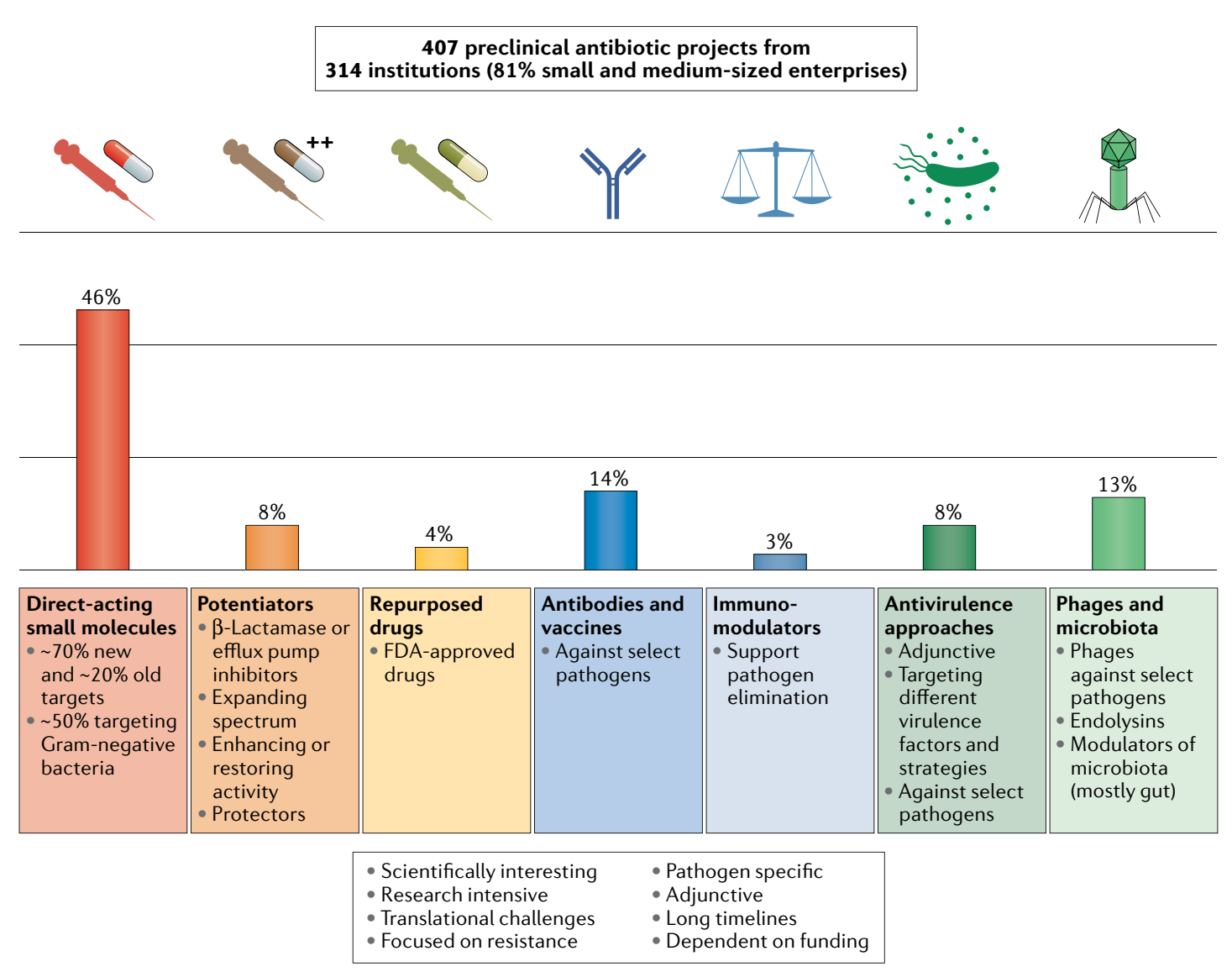

Fig. 1 | Overview of the preclinical antibacterial pipeline. We identified 314 research and development institutions and 407 preclinical projects. The projects were categorized according to their main effect on bacteria into the following groups: direct-acting agents, antibodies and vaccines, phages and phage-related products, microbiota-modulating therapies, antivirulence approaches, potentiators of direct-acting drugs, repurposed drugs, immunomodulators or others. The high diversity of approaches provided is innovative but carries high translational risks.

Formulations

Pharmaceutical formulation is the process in which the active compound and additional ingredients are combined to produce a final medicinal product.

\section{Parenteral application} Route of administration other than the gastrointestinal tract to achieve systemic distribution.

Intravesical application Administration of a drug directly into the bladder inhibit or kill bacteria directly but affect a broad range of virulence factors, 29 projects involve antibodies and antibody-drug conjugates, 27 projects involve antibacterial vaccines in preclinical development, 32 projects involve compounds that potentiate another drug, usually an existing antibiotic, 21 projects are studying microbiota-modulating approaches for different conditions, mostly focused on the gut microbiota, 15 projects are ongoing for repurposed non-antibiotics or antibiotics repurposed in combinations or developed for different fields or applications, 12 projects are aiming to modulate the immune system to support the elimination of pathogens and 18 projects are pursuing other strategies (for example, nanoparticles). Almost $40 \%$ of the projects are focused on pathogen-specific approaches, which is unprecedented in antibiotic history.

The discovery phases (hit-to-lead and lead optimization phases ending with declaration of a preclinical candidate) and preclinical development phases (clinical trial authorization (CTA) or investigational new drug application (IND), often called 'CTA/IND-enabling studies') are relatively evenly distributed and show a steady flow towards first-in-human studies. The geographical distribution across development phases is shown in FIG. 3b.

In the discovery phases, potential indications are often not decided yet as they depend on the achieved spectrum of the compound. Therefore, we applied general terms for indications (FIG. 3c), such as infections caused by Gram-negative bacteria, or infections caused by Gram-positive bacteria, mostly skin and soft tissue infections. Other indications include infections caused by Neisseria gonorrhoeae, Helicobacter pylori and Salmonella species.

Most new therapies will be formulated for parenteral application (mostly intravenous) (FIG. 3d). Vaccines with intramuscular application are included in this group. Agents with both intravenous and oral formulations were rare, and oral application was planned in only $10 \%$ of projects. Formulations for local administration include oral non-absorbable compounds and intravesical application. Inhalation is planned for 29 drugs (7\%). Local administration may avoid pharmacokinetic and/or toxicological challenges of systemic drug exposures.

Direct-acting agents. One hundred and eighty-seven projects involve traditional antibacterial agents that directly target bacteria by inhibiting or killing them without requiring any additional therapy. Characteristically, these compounds are synthesized or natural chemicals of mostly small size and they follow a traditional, well-known regulatory development pathway. These 
a All institutions

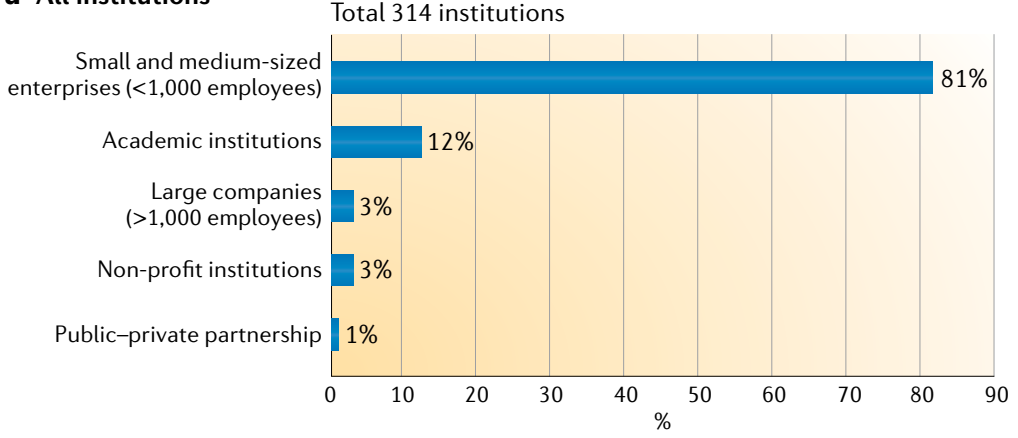

b Small and medium-sized enterprises

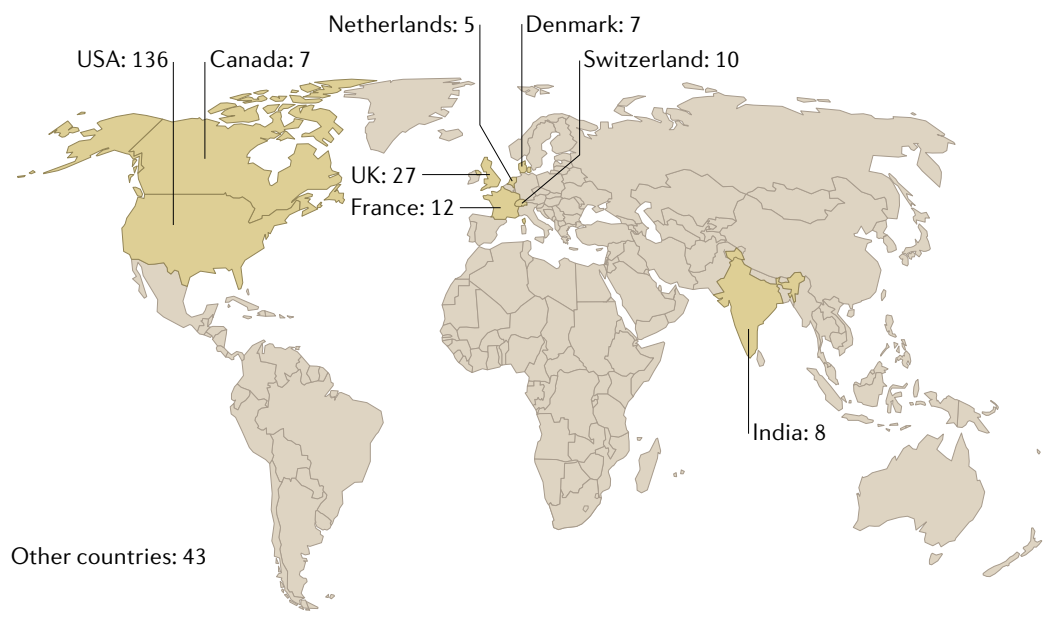

Fig. 2 | Type and location of institutions that carry out preclinical antibacterial development. a | The large majority of institutions involved in the preclinical discovery and preclinical development of antibacterials are small and medium-sized enterprises (255 of 314 institutions in total). Academic institutions, large companies, non-profit institutions and public-private partnerships are comparatively under-represented. b | More than half of the small and medium-sized enterprises are located in North America, followed by Europe as the second most prominent continent. The European countries with five or more companies are the United Kingdom, France, Switzerland, Denmark and the Netherlands.

\footnotetext{
Non-fermenters

Heterogeneous group of bacteria which cannot use glucose and thus are unable to generate energy through fermentation of glucose. Important genera of nonfermenters include Pseudomonas and Acinetobacter

Phase III trials In clinical development, phase III clinical trials are randomized controlled multicentre studies that assess the effectiveness and safety of a drug in comparison to current standard-of-care treatment.
}

direct-acting drugs can be further classified into three groups: improved derivatives of known antibiotic classes (old targets), new chemical classes with new targets and unknown or undefined agents with unclear targets (FIG. 4a). The group of old targets $(n=35,19 \%)$ includes $\beta$-lactams and other inhibitors of penicillin-binding proteins, fluoroquinolones and novel bacterial topoisomerase inhibitors, aminoglycosides, polymyxins and macrolides ${ }^{16}$. One hundred and thirty-five projects (72\%) are focused on new targets, including synthetic and natural antimicrobial peptides (AMPs), natural products and LpxC inhibitors (as discussed below). Other new targets include new binding sites in the bacterial ribosome, the membrane, the cell wall, transcription and/or translation, gene interference and metabolism ${ }^{17-20}$. Some of these targets and scaffolds were described a long time ago but were not pursued to clinical development. Seventeen projects involving direct-acting agents could not be grouped due to insufficient information. Almost half of the projects are focused broadly on Gram-negative bacteria (enterobacteria and non-fermenters), and $~ 10 \%$ are focused on Gram-positive bacteria (mostly staphylococci), which are not a critical priority according to the WHO priority list ${ }^{21}$. The cell wall of Gram-negative bacteria is an effective barrier to molecules that need to penetrate the outer and inner membranes. Therefore, the scientific challenges for targets residing in the cytoplasm or inner membrane are greater than those for novel targets located in the periplasm or in the outer membrane. Not surprisingly, there is a noticeable trend towards targets in the outer membrane in preclinical projects. As mentioned before, in early drug discovery the spectrum of activity cannot be defined exactly and may change during the lead optimization phase. About $22 \%$ of the projects involve pathogen-specific approaches (mostly against Gram-negative bacteria) and thus face specific challenges to recruit enough patients for phase III trials compared with trials of drugs with a broader spectrum. About $10 \%$ of the projects have a broad spectrum covering a broad range of both Gram-positive bacteria and Gram-negative bacteria (FIG. 4b).

Within the group of direct-acting compounds, three defined clusters are noticeable: synthetic or natural AMPs, natural products and LpxC inhibitors. The naturally abundant and diverse AMPs are a well-known group of antibacterials and are the basis for semisynthetic peptide molecules and peptidomimetics ${ }^{1822-25}$. Such renewed interest in this group may help to overcome some of the obstacles of AMPs such as high cost of synthesis, short half-life in vivo due to their susceptibility to proteolytic degradation and issues with toxicity ${ }^{26}$. Natural products are mainstays of our current antibiotic arsenal, exemplified by the large group of $\beta$-lactam antibiotics, aminoglycosides, tetracyclines and macrolides. Modern technologies such as genome mining contribute to the discovery of new scaffolds, and technical innovations are revealing new chemistry and increased yields, all of which contribute to the revival of natural product programmes ${ }^{27-29}$. LpxC inhibitors, which target the first dedicated step in the synthesis of lipid A, have been explored since the mid-1990s but no drug has advanced yet beyond phase I clinical trials. Development of ACHN-975 was discontinued after a phase I trial, owing to local inflammation at the injection site and some toxicity signals in the mouse model ${ }^{30-32}$. A trial involving RC-01, another LpxC inhibitor, was recently terminated for safety reasons ${ }^{33}$. Despite LpxC being a good target, toxicity of the used chemical matter seems to be a major challenge, but a growing body of knowledge and experience may help to overcome some of the current hurdles, including recent donations facilitated by CARB-X of toxicology data on the recently failed LpxC inhibitor into the public domain (the Shared Platform for Antibiotic Research and Knowledge (SPARK), Pew Trusts). In general, novel targets or novel chemicals carry the risk of unpredictable toxicity, because the translatability of safety signals from preclinical models to humans is uncertain, as exemplified by the aforementioned LpxC inhibitor RC-01 (REF. ${ }^{32}$ ). The recent termination of the phase III clinical trial of the novel Pseudomonas aeruginosa-specific LptD inhibitor murepavadin due to higher than expected rates of acute kidney injury demonstrates the challenges of unexpected toxicity of a new chemical that was not predicted from earlier preclinical studies or from studies in healthy individuals ${ }^{34}$. 
Metallo- $\beta$-lactamases

$\beta$-Lactamases that require zinc for activity and hydrolyse penicillins, cephalosporins and carbapenems.
Potentiators. Potentiators are drugs that have no or insufficient antibacterial activity alone but transform, restore or augment the activity of another antibiotic. Well-known examples include $\beta$-lactamase inhibitors ${ }^{35,36}$; of note, there are no approved inhibitors that include metallo- $\beta$-lactamases ${ }^{37}$. Twelve projects are focused on inhibiting $\beta$-lactamases, including metallo- $\beta$-lactamases (Supplementary Fig. 2). Some of the $\beta$-lactamase inhibitors are planned to be delivered orally. Although extensively researched, no inhibitor of various efflux pumps $s^{38}$ has been clinically developed so far ${ }^{39}$. Five efflux inhibitors targeting different efflux pumps are included in this list of potentiators. Other approaches in the preclinical pipeline are potentiators that expand the spectrum (for example, developing Gram-negative activity from antiGram-positive drugs), enhance the activity substantially, restore the activity against resistant bacteria or protect against nephrotoxicity of nephrotoxic antibiotics, such as colistin or aminoglycosides.

Repurposed drugs. We identified 15 projects involving repurposed drugs. Repurposed drugs are drugs that are approved for other disease areas or antibacterial drugs that have not been tested or not used for a specific purpose before. They could be developed in combination, as drug conjugates or in new formulations that allow a

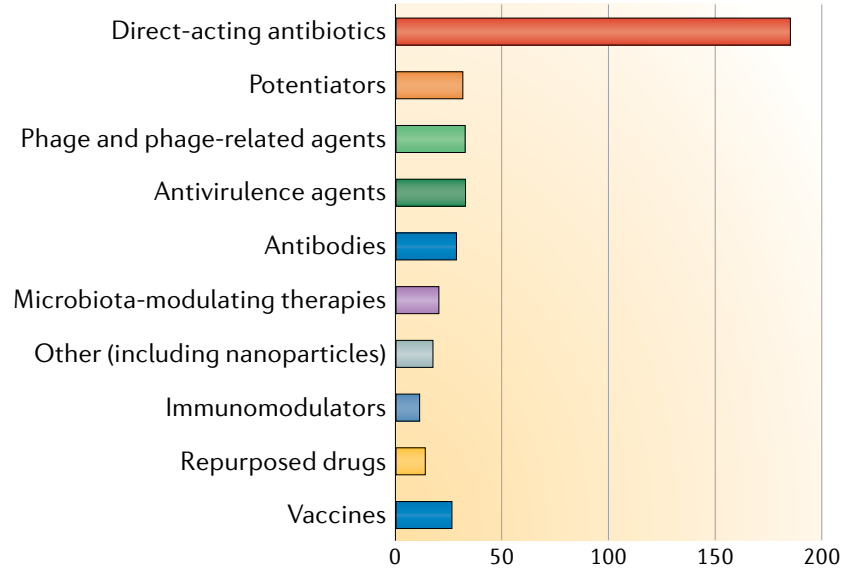

Infection with Gram-negative bacteria

Infection with Gram-positive bacteria

Cystic fibrosis

Clostridioides difficile infection

Urinary tract infection

Hospital-acquired/ventilator-

associated pneumonia

Tuberculosis

Implant-associated infection

Respiratory tract infection

Other

Undefined
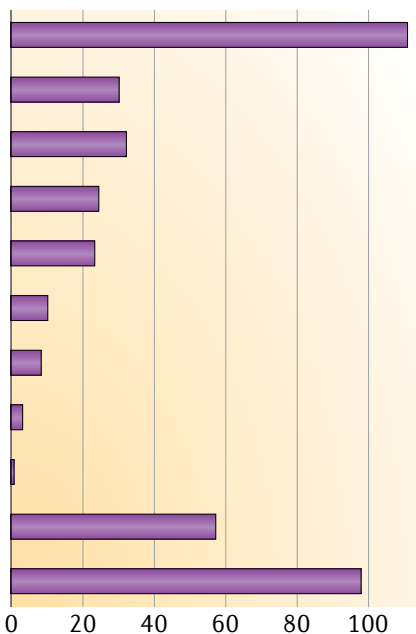

120 b

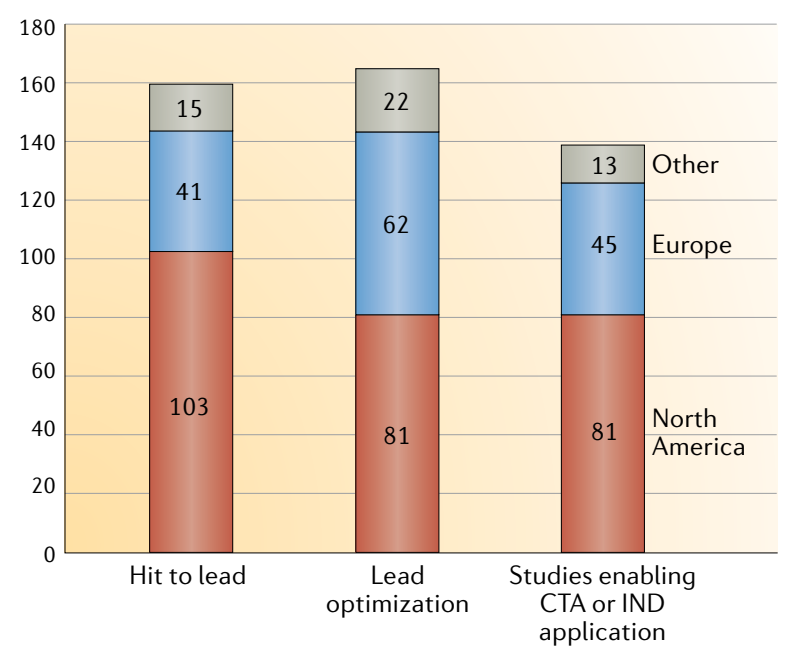

d

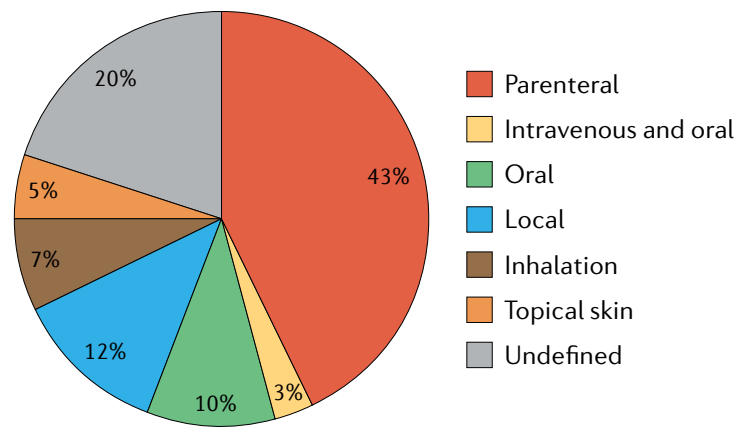

authorization (CTA)- and investigational new drug application (IND)-enabling studies with a trend towards relatively more projects in the early phase in North America and more projects in the later phases in Europe. c|Although the planned indications cannot be defined for all preclinical projects, the ones that have a planned indication already reflect the $\mathrm{WHO}$ priority list of pathogens for which new antibiotics are needed, such as infections with no or few available treatment options and that currently cause substantial morbidity and death, and/or are difficult to treat. $\mathbf{d}$ | Most of the agents for which the route of administration has already been defined will be applied parenterally (mostly intravenously and in case of vaccines also intramuscularly). Fewer projects will use oral administration (for systemic treatment, in a few projects this is combined with intravenous treatment), inhalation, local administration (mostly non-absorbable oral administration) and topical formulation for the skin. 


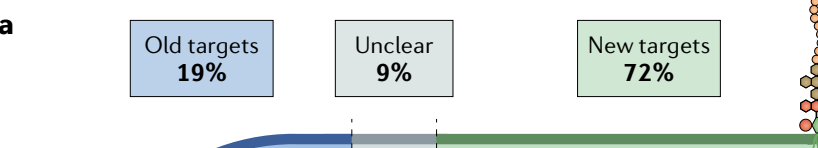

Polym

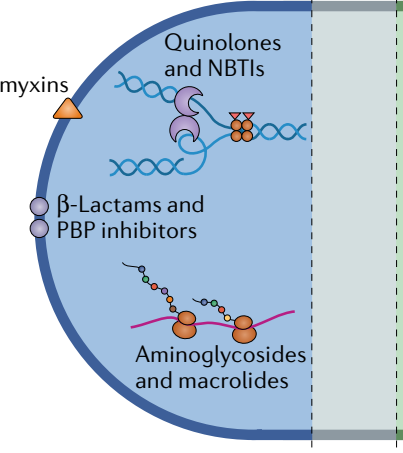

b

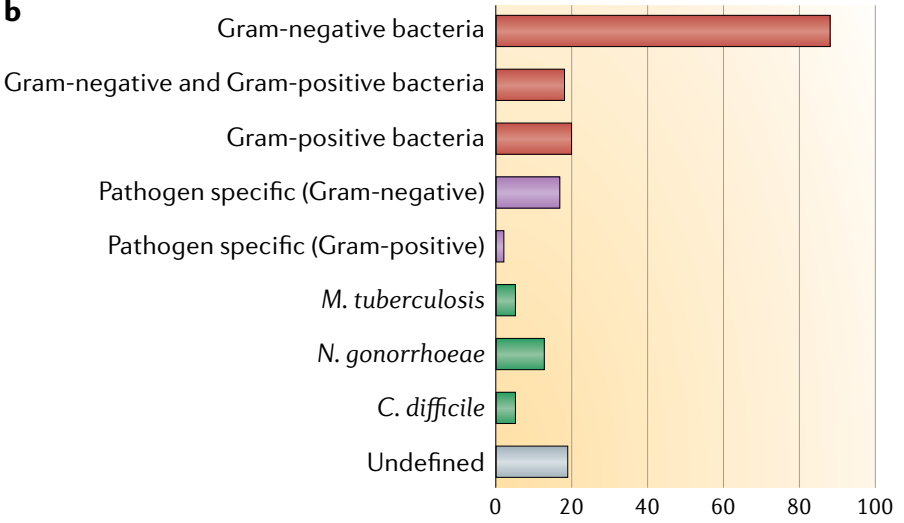

Fig. 4 | Approaches and spectrum of preclinical direct-acting, small-molecule antibacterials. a | Direct-acting small molecules in the preclinical antibiotic pipeline are derivatives of 'old chemical classes'. This group includes $\beta$-lactams and other penicillinbinding protein (PBP) inhibitors, fluoroquinolones, novel bacterial topoisomerase inhibitors (NBTIs), aminoglycosides, polymyxins and macrolides. Most direct-acting antibacterials represent new chemical classes and/or have new targets. These small molecules include large groups of synthetic and natural antimicrobial peptides, natural products and inhibitors of $\mathrm{LpxC}$, the first dedicated enzyme in lipid A synthesis. $\mathbf{b} \mid$ Most direct-acting small molecules target Gram-negative bacteria (either with a broader Gram-negative spectrum or pathogen specific). There are fewer molecules aimed at Gram-positive bacteria or with a broad spectrum against both Gram-negative bacteria and Gram-positive bacteria. The numbers for Neisseria gonorrhoeae, Clostridioides difficile and Mycobacterium tuberculosis are shown separately.

\section{Endolysins}

Enzymes that are produced by bacteriophages and hydrolyse the bacterial cell wall to escape the cell at the end of the cycle.

Compassionate use The use of unapproved drugs outside clinical trials for patients without options of treatment with an approved drug. different use $\mathrm{e}^{12,40}$. The development process for repurposed drugs benefits from a large body of available knowledge and reduces the time and cost of development $^{12}$. The value of such an approach in the clinical setting remains to be shown.

Phage and phage-derived peptides. Twenty-seven institutions are working on the development of 33 phage or phage-derived therapeutics. Phage therapies may contain natural phage cocktails (11 projects), engineered phage cocktails (11 projects, some CRISPR enhanced) and other highly diverse scientific approaches (FIG. 5a). The most common phage-derived products are phage endolysins against Staphylococcus aureus, with relatively fewer projects on recombinant lysins against Gramnegative bacteria. Phage therapies are species specific and thus the most common targets of the programmes were $P$. aeruginosa and $S$. aureus, but phage therapies for Clostridioides difficile infection and infection with a wide range of other pathogens are also in development (FIG. 5b).

Phage therapies have garnered a lot of attention lately due to the successful treatment of a small number of individual patients with chronic conditions limited to a small number of experimental treatment centres, often in compassionate use programmes ${ }^{10,41,42}$. Compassionate use of personalized phage preparations is limited to specific clinical circumstances and individual physicians and researchers who have experience with phage ther$\mathrm{apy}^{42}$. Patient-specific phage cocktails allow the use for rare pathogens, whereas recombinant lysins may cover a broader spectrum of Gram-negative bacteria. Although phages have been used historically in topical formulations (mostly skin) ${ }^{43}$, phage preparations are being developed for intravenous, aerosol or diverse locally applied formulations $s^{44}$. The immense size of phages compared with small-molecule antibiotics poses pharmacokinetic challenges, and important scientific questions remain regarding availability at the site of infection and determining the best dosing regimen ${ }^{45}$. In general, natural and engineered phage cocktails dominate our sample. New genetic tools such as CRISPR-Cas systems are used to genetically engineer phages that infect diverse hosts ${ }^{46}$. Phages are also used as species-specific carriers for a variety of potential antibacterial payloads ${ }^{47}$ or CRISPR-Cas-based RNA-guided nucleases targeted at resistance or virulence determinants ${ }^{48,49}$. Considerable progress has been made recently in tackling the great challenges in the chemistry, manufacturing and control of therapeutic phages, especially in production, stability, purity and quality control. However, challenges remain, such as unique phage biology and specificity, pharmacokinetics of large self-replicating agents, rapid resistance development and translation to a broader group of patients beyond compassionate use ${ }^{8}$. Also, patientspecific phage therapy requires a highly developed diagnostic infrastructure (with phage-specific rapid testing). Phage therapies will likely be restricted to well-defined situations in individual patients or as adjunctive therapy with all the challenges related to clinical superiority trials that compare a usually highly effective standard of care and adjunctive therapy versus standard of care alone $e^{50,51}$.

Phage-derived proteins such as endolysins are gaining attention ${ }^{52,53}$. Endolysins are bacteriolytic on contact and are highly specific for a bacterial species or genus. Endolysins directed against $S$. aureus are in clinical development ${ }^{8}$ and follow the traditional clinical development path. Extensive protein engineering efforts have expanded options to target Gram-negative bacteria ${ }^{54}$. However, such projects are still uncommon and may require more basic research ${ }^{55}$.

Microbiota-modulating therapies. Twenty-one different microbiota-modulating approaches are included in this Review (FIC. 5c). The most common strategy is engineered probiotics (also called live biotherapeutic products') with potentially enhanced functional properties. Other projects are focused on natural strains derived from a healthy microbiota for a variety of potential beneficial effects. AMPs expressed in phage-based carrier 
a
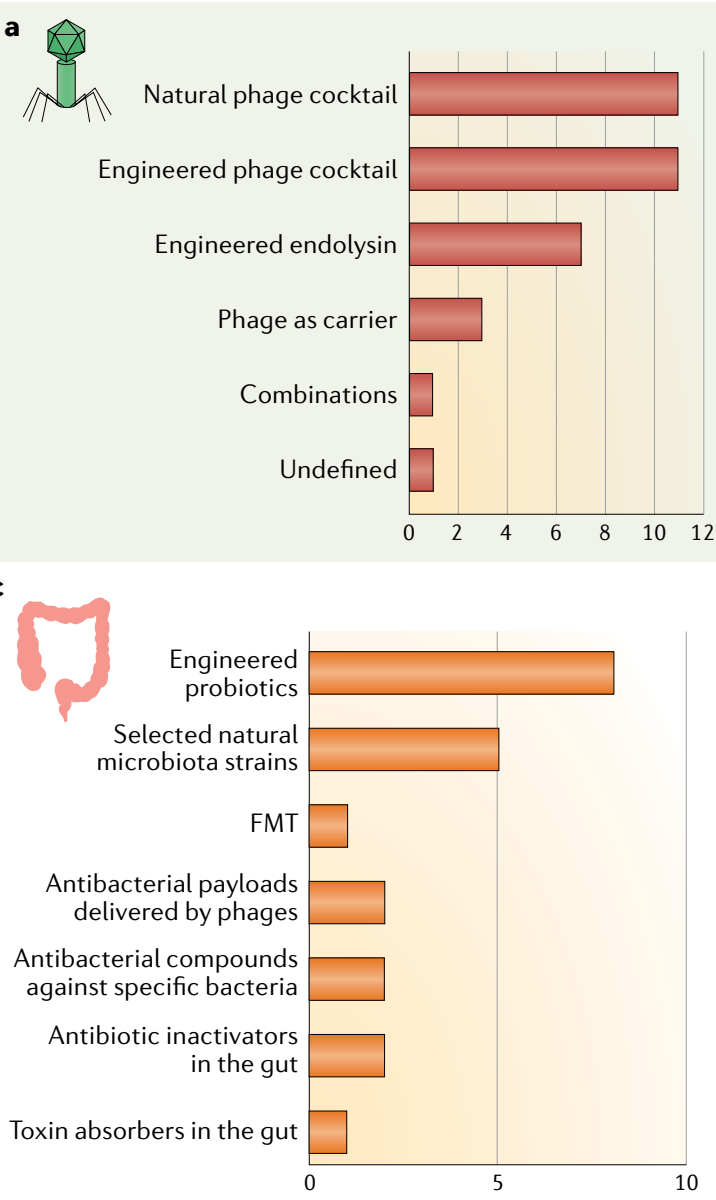

b
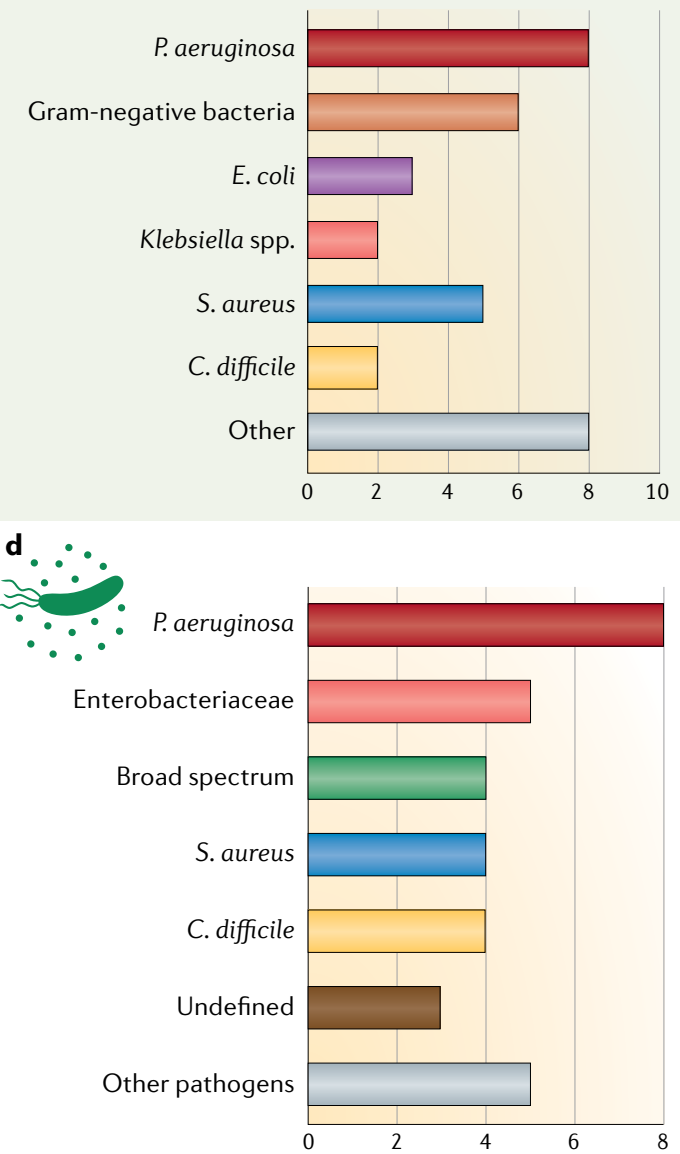

Fig. 5 | Phages and phage-derived therapeutics, microbiota-modulating approaches and antivirulence approaches in the preclinical pipeline. a| The most commonly pursued approaches for phage therapy are phage cocktails (either natural phages or engineered phages), engineered phage endolysins and phages that are used as carriers for antibacterial payloads. $\mathbf{b} \mid$ The phages and phage-derived proteins in preclinical development target a large variety of different pathogens and are usually pathogen specific. c| Microbiota-modulating therapies are mainly engineered probiotics or selected natural bacterial strains from a healthy microbiota. We have also included phages as a carrier of antibacterial payloads that specifically manipulate the microbiota in this category. Other microbiota-modulating approaches include use of antibacterial compounds against specific bacteria in the microbiota, antibiotic inactivators in the gut and absorbers of bacterial toxins in the gut, and faecal microbiota transplants (FMT). $\mathbf{d}$ | The spectrum of antivirulence compounds is diverse and focuses on Pseudomonas aeruginosa, Enterobacteriaceae spp., Staphylococcus aureus and Clostridioides difficile or, less commonly, is broad.

systems are another approach to modulate the microbiota by targeting specific members of the microbiota. Antibiotic inactivators and absorbers of bacterial toxins in the gut are also being pursued in preclinical projects. Most microbiota-modulating therapies in preclinical development target the gut microbiota, especially C. difficile. The lung, sinus or skin microbiota is rarely a target of such approaches.

Recent advances in metagenomic, computational and synthetic biology tools have allowed and inspired the revival of research into the human microbiota ${ }^{56}$. Microbiota-modifying therapies have been explored and tested in patients using the entire healthy microbiota to correct major imbalances and reduce the recurrence of C. difficile infection ${ }^{57}$. Such programmes have recently faced a setback as one patient died because of faecal transplants that contained drug-resistant bacteria ${ }^{58}$ and led to the halting of clinical trials by the FDA. It is not fully known yet how this incident will affect regulation by the FDA and consequently the entire field of faecal transplants or similar strategies. There is a trend towards reducing the complexity of faecal transplants by controlling the transfer of bacterial strains or selecting natural strains derived from a healthy microbiota ${ }^{59,60}$. New techniques based on synthetic biology and systems biology allow the precise genetic engineering of well-known probiotics ${ }^{61}$, which may also express specific antibacterial substances ${ }^{62-64}$. A long-known strategy to maintain a healthy microbiota is the use of antibiotic inactivators or absorbers of bacterial toxins in the gut. Examples include enzymes that inactivate residues of specific systemic antibiotics in the gut to reduce disbalance of the microbiota caused by antibiotic therapy or absorbers of bacterial toxins that may cause disease, such as toxins produced by $C$. difficile or other pathogens ${ }^{64,65}$. Both strategies still need to prove their value in the clinical situation ${ }^{51}$. 
The drastically reduced complexity of new therapies based on genetic engineering technologies but incomplete knowledge of the microbiota may hamper the translation to an effective modulation of an extremely complex system. On the other hand, highly synthetic strategies reduce or avoid the risk of transferring potentially unwanted bacteria or other components of the microbiota $^{66}$. The challenges of microbiota-modifying therapies are even more obvious when targeting bacterial communities beyond the gut microbiota. Validated animal models to predict clinical outcome are lacking. The entire microbiota field has seen great attention in terms of investment and company formation, with a potentially overly optimistic promise to cure a wide variety of diseases and generate high profits. In the infectious disease field, we see some spillover of this enthusiasm.

Antivirulence therapies. The 33 antivirulence projects that we identified are pursuing a wide range of strategies, including inhibition of quorum sensing, biofilm formation, adhesion, diverse regulators and persisters $^{67-71}$. Antivirulence drugs need to be combined with a direct-acting antibacterial therapeutic and are designed as adjunctive therapies. Most programmes are specifically targeted at $P$. aeruginosa, $S$. aureus and C. difficile. Some approaches target several members of the Enterobacteriaceae family or have an even broader spectrum (FIG. 5d).

The discovery phase of antivirulence therapies is characterized by the difficult choice of the most relevant preclinical assays to define success in the absence of bacterial death ${ }^{8,72}$. As surrogate outcomes may have little evidence of relevance for clinical outcome, the risk of failure in clinical trials is high. Validated animal models that would predict clinical outcome are usually not available.

Additionally, many antivirulence programmes ${ }^{73}$, similarly to phage therapies, are pathogen-specific and often patient-specific approaches. They would require not only advanced health-care systems but also specific diagnostic capabilities that are beyond the currently available and implemented ones, and there are few or no plans to ensure timely development and deployment of diagnostic tools to guide the potential clinical use of new antivirulence therapies.

Antibodies and antibody-drug conjugates. Twenty-nine projects are focused on antibodies, including antibodydrug conjugates. Most antibodies are developed as prevention or adjunctive therapy for $S$. aureus infections, followed by $C$. difficile and $P$. aeruginosa infections, with more than three programmes each. Less common are antibodies against Acinetobacter species, Escherichia coli and other bacteria. Eleven of these programmes are already in late preclinical development.

Only three antibodies against bacterial infections have been approved for clinical use so far ${ }^{8}$. They are active against toxins of Clostridium botulinum, Bacillus anthracis and C. difficile. All of these approved antibodies neutralize toxins, the predominant or the only virulence factor responsible for diseases caused by these pathogens. Antibodies against bacteria that have a multitude of virulence determinants have yet to be successful. The recent clinical failure of an antibody against several virulence factors of $S$. aureus ${ }^{74}$ exemplifies the challenges of conducting superiority trials and showing efficacy and clinical value of an adjunctive therapy. Similarly, it is extremely difficult to show a meaningful clinical benefit when administering antibodies prophylactically. For example, even in groups at high risk of postoperative $S$. aureus infection, the number of infections is small, and therefore large numbers of enrolled patients are needed to make an overall effect visible in clinical trials.

Vaccines. Among the 27 vaccine projects, five target $S$. aureus. Fewer than five projects are targeting P. aeruginosa, Acinetobacter species, Klebsiella pneumoniae, N. gonorrhoeae and non-typhoidal Salmonella spp. Several other projects are focused on single rare pathogens. There are also multiantigen and/or multivalent vaccines against groups of bacteria.

Some of the aforementioned challenges regarding antibodies also apply to vaccines in development to prevent infections caused by multidrug-resistant pathogens. Several bacterial vaccine trials have failed in late clinical development owing to the lack of reliable preclinical predictive models followed by insufficient clinical efficacy confounded by concurrent antibiotic treatment ${ }^{75,76}$. Most targeted pathogens for current vaccine projects have been classified as less well suited to vaccine development or as having unclear development feasibility by a recent report that evaluated research and development opportunities for vaccines ${ }^{9}$.

Other projects. This group of 18 projects includes nanoparticles (nanobiotics) that have antibacterial capabilities. While nanoparticles and synthetic polymers are well-known vectors to deliver drugs ${ }^{77,78}$, nanobiotics are able to kill microorganisms directly through the generation of reactive oxygen species, cell membrane permeation, triggering DNA damage or interrupting transmembrane electron transport ${ }^{79}$.

\section{Conclusions}

The preclinical antibacterial pipeline (BOX 2) reveals innovative strategies when contrasted with the current global antibacterial clinical pipeline, which mainly builds on modification of known antibiotic classes. The preclinical pipeline is characterized by a high level of diversity and interesting scientific concepts compared with the clinical pipeline, although these projects may not necessarily contribute to solving the problem of increasing resistance to currently available antibiotics. The focus and goal of most of the current projects acknowledge the need for new therapies without cross-resistance to existing antibiotics. In sharp contrast to the clinical pipeline, more than $70 \%$ of the direct-acting agents are new classes, have new targets or have new mechanisms of action not used so far in patients. Most of these 'new' approaches were described decades ago but were not followed through to clinical development.

The general goal and focus of preclinical development programmes on Gram-negative pathogens correspond to the need described in the WHO priority pathogen list ${ }^{21}$. 


\section{Box 2 | Main features of the preclinical antibacterial pipeline}

- High level of diversity and interesting scientific approaches, much more so than the clinical pipeline.

- Less than half of the projects involve direct-acting small molecules.

- More than half of the projects involve 'non-traditional', potentially adjunctive therapies with an as yet unclear regulatory pathway to show a clinically relevant benefit.

- Non-traditional approaches may not build on validated predictive preclinical models and therefore have a higher risk of clinical failure.

- Focus on WHO critical priority pathogens (with the exception of antibodies, vaccines and phages for Staphylococcus aureus).

- Strong trend towards pathogen-specific or patient-specific therapy requiring highly developed health-care systems with advanced rapid diagnostic capabilities.

- Strong dependence on public and/or philanthropic funding.

- High volatility due to high-risk strategies and translational challenges pursued by small companies.
Innovative readouts or biomarkers have not been developed yet to measure the impact of these therapies. In addition to the challenges mentioned, some approaches require a delivery system to transport active agents to the site of action, thus representing the challenge of two new complex systems. Translating scientific results and non-clinical studies into significant clinical benefits will be the greatest challenge for most indirect-acting non-traditional therapies.

Although this Review is the most complete analysis and up-to-date description of the global antibacterial preclinical research and development pipeline, some limitations apply. Certain sectors, such as academic or non-profit institutions, may be underrepresented in the data, whereas other institutions may abandon their discovery activities by closing the company or project before any public disclosure. Some of our data sources and programmes (REPAIR Impact Fund, ENABLE and Joint Programming Initiative on Antimicrobial Resistance) were limited to specific regions, mainly North America and Europe. Although CARB-X accepts applications from around the world, most of its applications are from North America and Europe, and some institutions outside those regions may not have applied for funding. Most of the data do not systematically include tuberculosis, so those programmes are probably underrepresented.

In conclusion, the preclinical development pipelines are diverse and innovative compared with the clinical pipeline, although this innovation does not necessarily solve the most critical therapeutic problems and may not translate to relevant clinical effects. The pipelines are highly fragile in SMEs for many reasons. Major basic scientific challenges such as penetration, efflux and the associated risk of toxicity owing to required high doses need an expanded concerted research agenda to advance discovery efforts for traditional antibiotics. A large proportion of high-risk approaches have yet unknown ability to translate into clinical beneficial potential, which may reduce future viable clinical pipelines. Basic scientific and translational challenges cannot be solved by individual small companies and will require huge collaborative efforts of the entire drug discovery community. Clear clinical development strategies may not exist for some non-traditional approaches. Adjunctive therapies require an active antibacterial drug and thus may not solve the current resistance problem. A strong focus on narrow-spectrum, pathogen-specific and patientspecific therapies will require highly developed and welldeployed diagnostics. These are not yet in view and may be restricted to specific countries and environments, primarily better-resourced environments. This Review further reveals the overlap between the preclinical pipeline and the WHO priority pathogen list and the scope of some funding calls that currently include specifically high-risk approaches with unsolved translational challenges. A global public health perspective would improve the potential medical value of future treatments. Many antibacterial projects are scientifically exciting and innovative but as the translational challenges are extremely great and most preclinical projects will fail to result in approved and clinically relevant drugs, the preclinical 
Push funding

Incentivizes discovery and development activities before achieving regulatory approval.

Pull incentives

Reward the successful development and regulatory approval of a new drug. pipeline is not adequately robust. Owing to numerous discovery challenges, it is not surprising to find the global antibacterial clinical pipeline populated with modifications of existing classes of antibiotics that substantially de-risk programmes. Additionally, the dearth of funding available for clinical development of antibacterial therapies contributes to barriers for progression of preclinical projects to human trials after all the scientific challenges in the preclinical phase have been overcome. A long-term commitment of sustained push funding, pull incentives and new concepts for commercializing and delivering future therapies will be necessary to ensure that current projects will potentially benefit society in the future.

Published online 19 November 2019
1. Lobanovska, M. \& Pilla, G. Penicillin's discovery and antibiotic resistance: lessons for the future? Yale J. Biol. Med. 90, 135-145 (2017).

2. Aminov, R. I. A brief history of the antibiotic era: lessons learned and challenges for the future. Front. Microbiol. 1, 134-134 (2010).

3. Podolsky, S. H. The antibiotic era: reform, resistance, and the pursuit of a rational therapeutics. (John Hopkins University Press, 2015).

4. Baker, S. J., Payne, D. J., Rappuoli, R. \& De Gregorio, E. Technologies to address antimicrobial resistance. Proc. Natl Acad. Sci. USA 115, 12887-12895 (2018) Extensive review of past antibacterial therapies and opportunities for future strategies to provide new therapies.

5. Cars, O. Securing access to effective antibiotics for current and future generations. Whose responsibility? Ups J. Med. Sci. 119, 209-214 (2014).

6. Interagency Coordination Group on Antimicrobial Resistance. No time to wait: securing the future from drug-resistant infections. Report to the SecretaryGeneral of the United Nations. https://www.who.int antimicrobial-resistance/interagency-coordinationgroup/IACG_final_report_EN.pdf (2019).

7. Theuretzbacher, U. et al. Analysis of the clinical antibacterial and antituberculosis pipeline. Lancet Infect. Dis. 19, e40-e50 (2019).

8. Theuretzbacher, U. \& Piddock, L. J. V. Non-traditional antibacterial therapeutic options and challenges. Cell Host Microbe 26, 61-72 (2019).

9. Wellcome Trust $\&$ The Boston Consulting Group. Vaccines to tackle drug resistant infections. An evaluation of $R \& D$ opportunities, https:// vaccinesforamr.org (2019).

10. Kortright, K. E., Chan, B. K., Koff, J. L. \& Turner, P. E. Phage therapy: a renewed approach to combat antibiotic-resistant bacteria. Cell Host Microbe 25 219-232 (2019)

11. Motley, M. P. \& Fries, B. C. A new take on an old remedy: generating antibodies against multidrugresistant gram-negative bacteria in a postantibiotic world. mSphere 2, e00397-00317 (2017).

12. Mirō-Canturri, A., Ayerbe-Algaba, R. ¿ Smani, Y. Drug repurposing for the treatment of bacterial and fungal infections. Front Microbiol. 10, 41 (2019).

13. Rello, J., Parisella, F. R. \& Perez, A. Alternatives to antibiotics in an era of difficult-to-treat resistance: new insights. Expert Rev. Clin. Pharmacol. 12, 635-642 (2019)

14. Monserrat-Martinez, A., Gambin, Y. \& Sierecki, E. Thinking outside the bug: molecular targets and strategies to overcome antibiotic resistance. Int. J. Mol. Sci. 20, E1255 (2019). An extensive overview of past and current strategies for antibacterial therapies.

15. Domalaon, R., Idowu, T., Zhanel, G. G. \& Schweizer, F. Antibiotic hybrids: the next generation of agents and adjuvants against gram-negative pathogens? Clin. Microbiol. Rev. 31, e00077-17 (2018).

16. Kohanski, M. A., Dwyer, D. J. \& Collins, J. J. How antibiotics kill bacteria: from targets to networks Nat. Rev. Microbiol. 8, 423-435 (2010).

17. Lin, J., Zhou, D., Steitz, T. A., Polikanov, Y. S. \& Gagnon, M. G. Ribosome-targeting antibiotics: modes of action, mechanisms of resistance, and implications for drug design. Annu. Rev. Biochem. 87, 451-478 (2018)

18. Ciumac, D., Gong, H., Hu, X. \& Lu, J. R. Membrane targeting cationic antimicrobial peptides. J. Colloid. Interface Sci. 537, 163-185 (2019).

19. Ma, C., Yang, X. \& Lewis, P. J. Bacterial transcription as a target for antibacterial drug development. Microbiol. Mol. Biol. Rev. 80, 139-160 (2016).

20. Silver, L. L. Appropriate targets for antibacteria drugs. Cold Spring Harb. Perspect. Med. 6, a030239 (2016).
21. Tacconelli, E. et al. Discovery, research, and development of new antibiotics: the WHO priority list of antibiotic-resistant bacteria and tuberculosis. Lancet Infect. Dis. 18, 318-327 (2018). The WHO list prioritizes target pathogens for antibacterial drug research and discovery with a global perspective.

22. Kang, H.-K., Kim, C., Seo, C. H. \& Park, Y. The therapeutic applications of antimicrobial peptides (AMPs): a patent review. J. Microbiol. 55, 1-12 (2017).

23. Molchanova, N., Hansen, P. R. \& Franzyk, H. Advances in development of antimicrobial peptidomimetics as potential drugs. Molecules 22, 1430 (2017).

24. Hoda, M. et al. Antimicrobial peptides: features, action, and their resistance mechanisms in bacteria. Microb. Drug Res. 24, 747-767 (2018)

25. Torres, M. D. T. Sothiselvam, S., Lu, T. K \& de la Fuente-Nunez, C. Peptide design principles for antimicrobial applications. J. Mol. Biol. 431 3547-3567 (2019).

26. Kuppusamy, R., Willcox, M., Black, D. S. \& Kumar, N. Short cationic peptidomimetic antimicrobials. Antibiotics 8, 44 (2019).

27. Wright, G. D. Opportunities for natural products in 21 st century antibiotic discovery. Nat. Prod. Rep. 34 , 694-701 (2017).

28. Rossiter, S. E., Fletcher, M. H. \& Wuest, W. M. Natural products as platforms to overcome antibiotic resistance. Chem. Rev. 117, 12415-12474 (2017).

29. Fedorenko, V. et al. Antibacterial discovery and development: from gene to product and back. Biomed Res. Int. 2015, 591349-591349 (2015).

30. Erwin, A. L. Antibacterial drug discovery targeting the lipopolysaccharide biosynthetic enzyme LpxC. Cold Spring Harb. Perspect. Med. 6, a025304 (2016).

31. Fang, L. \& Shutao, M. Recent process in the inhibitors of UDP-3-O-(R-3-hydroxyacyl)-nacetylglucosamine deacetylase (LpxC) against gram-negative bacteria Mini Rev. Med. Chem. 18, 310-323 (2018).

32. Cohen, F. et al. Optimization of LpxC inhibitors for antibacterial activity and cardiovascular safety. Chem. Med. Chem. 14, 1560-1572 (2019).

33. US National Library of Medicine. ClinicalTrials.gov, https://clinicaltrials.gov/ct2/show/NCT03832517 (2019).

34. Wach, A., Dembowsky, K. \& Dale, G. E. Pharmacokinetics and safety of intravenous murepavadin infusion in healthy adult subjects administered single and multiple ascending doses. Antimicrob. Agents Chemother. 62 , e02355-17 (2018)

35. Philippon, A., Jacquier, H., Ruppe, E. \& Labia, R. Structure-based classification of class a betalactamases, an update. Curr. Res. Transl. Med. S2452-3186, 30021-30022 (2019)

36. Bush, K. \& Bradford, P. A. Interplay between $\beta$-lactamases and new $\beta$-lactamase inhibitors. Nat. Rev. Microbiol. 17, 295-306 (2019).

37. Shi, C. et al. Approaches for the discovery of metallo$\beta$-lactamase inhibitors: a review. Chem. Biol. Drug Des. 94, 1427-1440 (2019)

38. Greene N. P., Kaplan, E Crow, A \& Koronakis, V. Antibiotic resistance mediated by the MacB ABC transporter family: a structural and functional perspective. Front Microb. 9, 950-950 (2018)

39. Lamut, A., Peterlin Mašič, L., Kikelj, D. \& Tomašič, T. Efflux pump inhibitors of clinically relevant multidrug resistant bacteria. Med. Res. Rev. https://doi.org/ 10.1002/med.21591 (2019)

40. Peyclit, L., Baron, S. A. \& Rolain, J.-M. Drug repurposing to fight colistin and carbapenem-resistant bacteria. Front Cell Infect. Microbiol. 9, 193-193 (2019).

41. Rohde, C., Wittmann, J. \& Kutter, E. Bacteriophages: a therapy concept against multi-drug-resistant bacteria. Surg Infect. 19, 737-744 (2018).
42. McCallin, S., Sacher, J. C., Zheng, J. \& Chan, B. K Current state of compassionate phage therapy. Viruses 11, 343 (2019).

43. Morozova, V. V., Vlassov, V. V. \& Tikunova, N. V. Applications of bacteriophages in the treatment of localized infections in humans. Front. Microbiol. 9 1696-1696 (2018).

44. Chang, R. Y. K. et al. Phage therapy for respiratory infections. Adv. Drug Deliv. Rev. 133, 76-86 (2018).

45. Dąbrowska, K. Phage therapy: what factors shape phage pharmacokinetics and bioavailability? Systematic and critical review. Med. Res. Rev, https:/ doi.org/10.1002/med.21572 (2019). Critical challenges in treating bacterial infections at different body sites are reviewed.

46. Hatoum-Aslan, A. Phage genetic engineering using CRISPR-Cas systems. Viruses 10, 335 (2018).

47. Dams, D., Brøndsted, L., Drulis-Kawa, Z \& Briers, Y. Engineering of receptor-binding proteins in bacteriophages and phage tail-like bacteriocins. Biochem. Soc. Trans. 47, 449-460 (2019).

48. Patsali, P., Kleanthous, M. \& Lederer, C. W. Disruptive technology: CRISPR/Cas-based tools and approaches. Mol. Diagn. Ther. 23, 187-200 (2019).

49. Vila, J. Microbiota transplantation and/or CRISPR/Cas in the battle against antimicrobial resistance. Clin Microbiol. Infect. 24, 684-686 (2018).

50. Segall, A. M., Roach, D. R. \& Strathdee, S. A. Stronger together? Perspectives on phage-antibiotic synergy in clinical applications of phage therapy. Curr. Opin. Microbiol. 51, 46-50 (2019).

51. Rex, J. H., Fernandez Lynch, H., Cohen, G., Darrow, J. J. \& Outterson, K. Designing development programs for non-traditional antibacterial agents. Nat. Commun. 10, 3416 (2019)

52. Fischetti, V. A. Development of phage lysins as novel therapeutics: a historical perspective. Viruses 10, 310 (2018).

53. Abdelkader, K., Gerstmans, H., Saafan, A., Dishisha, T. $\&$ Briers, Y. The preclinical and clinical progress of bacteriophages and their lytic enzymes: the parts are easier than the whole. Viruses 11, 96 (2019).

54. Oliveira, H., São-José, C. \& Azeredo, J. Phage-derived peptidoglycan degrading enzymes: challenges and future prospects for in vivo therapy. Viruses 10, 292 (2018)

55. Briers, Y. \& Lavigne, R. Breaking barriers: expansion of the use of endolysins as novel antibacterials against gram-negative bacteria. Future Microbiol. 10, 377-390 (2015)

56. Falony, G. et al. The human microbiome in health and disease: hype or hope. Acta Clin. Belg. 74, 53-64 (2019).

Recent gut microbiome research may help to accelerate translation of microbiome findings from bench to bedside.

57. Cammarota, G. et al. European consensus conference on faecal microbiota transplantation in clinical practice. Gut 66, 569-580 (2017).

58. FDA. FDA In Brief: FDA warns about potential risk of serious infections caused by multi-drug resistant organisms related to the investigational use of fecal microbiota for transplantation. https://www fda. gov/ news-events/fda-brief/fda-brief-fda-warns-aboutpotential-risk-serious-infections-caused-multidrug-resistant-organisms (2019).

59. Kellermayer, R. Fecal microbiota transplantation great potential with many challenges. Transl. Gastroenterol. Hepatol. 4, 40-40 (2019).

60. Iqbal, U., Anwar, H. \& Karim, M. A. Safety and efficacy of encapsulated fecal microbiota transplantation for recurrent clostridium difficile infection: a systematic review. Eur. J. Gastroenterol. Hepatol. 30, 730-734 (2018).

61. Mathipa, M. G. \& Thantsha, M. S. Probiotic engineering: towards development of robust probiotic strains with enhanced functional properties and for 
targeted control of enteric pathogens. Gut Pathog. 9, 28-28 (2017).

62. Ghosh, C., Sarkar, P., Issa, R. \& Haldar, J. Alternatives to conventional antibiotics in the era of antimicrobial resistance. Trends Microbiol. 27, 323-338 (2019).

63. Ozdemir, T., Fedorec, A. J. H., Danino, T. $\delta$ Barnes, C. P. Synthetic biology and engineered live biotherapeutics: toward increasing system complexity. Cell Syst. 7, 5-16 (2018).

64. Fehér, C., Soriano, A. \& Mensa, J. A review of experimental and off-label therapies for clostridium difficile infection. Infect. Dis. Ther 6, 1-35 (2017).

65. Connelly, S., Fanelli, B., Hasan, N. A., Colwell, R. R. \& Kaleko, M. Oral metallo-beta-lactamase protects the gut microbiome from carbapenem-mediated damage and reduces propagation of antibiotic resistance in pigs. Front Microbiol. 10, 101-101 (2019).

66. Ramachandran, G. \& Bikard, D. Editing the microbiome the CRISPR way. Philos. Trans. R. Soc Lond. B. Biol. Sci. 374, 20180103 (2019). New technologies bring challenges and opportunities for future new therapies that are reviewed in this article.

67. Pena, R. T. et al. Relationship between quorum sensing and secretion systems. Front Microbiol. 10 1100-1100 (2019).

68. Sharma, D., Misba, L. \& Khan, A. U. Antibiotics versus biofilm: an emerging battleground in microbial communities. Antimicrob. Resist. Infect. Control 8 76-76 (2019)

69. Murugayah, S. A. \& Gerth, M. L. Engineering quorum quenching enzymes: progress and perspectives. Biochem. Soc. Trans. 47, 793-800 (2019).

70. Defraine, V., Fauvart, M. \& Michiels, J. Fighting bacterial persistence: Current and emerging antipersister strategies and therapeutics. Drug Resist. Updat. 38, 12-26 (2018)

71. Fleitas Martínez, O., Cardoso, M. H., Ribeiro, S. M \& Franco, O. L. Recent advances in anti-virulence therapeutic strategies with a focus on dismantling bacterial membrane microdomains, toxin neutralization, quorum-sensing interference and biofilm inhibition. Front Cell Infect. Microbiol. 9, 74-74 (2019).

72. Maura, D., Ballok, A. E. \& Rahme, L. G. Considerations and caveats in anti-virulence drug development. Curr. Opin. Microbiol. 33, 41-46 (2016).

73. Dickey, S. W. Cheung G. Y. C. \& Otto, M. Different drugs for bad bugs: antivirulence strategies in the age of antibiotic resistance. Nat. Rev. Drug Discov. 16 , 457-471 (2017)

74. Aridis Pharmaceuticals, Inc. Aridis Pharmaceuticals reports phase 2 clinical trial results of AR-105 for the treatment of ventilator-associated pneumonia caused by Pseudomonas Aeruginosa Investors aridispharma. com. https://investors.aridispharma.com/2019-0903-Aridis-Pharmaceuticals-Reports-Phase-2-ClinicalTrial-Results-of-AR-105-for-the-Treatment-

of-Ventilator-Associated-Pneumonia-Caused-byPseudomonas-Aeruginosa (2019)

75. Redi, D., Raffaelli, C. S., Rossetti, B., De Luca, A. \& Montagnani, F. Staphylococcus aureus vaccine preclinical and clinical development: current state of the art. New Microbiol. 41, 208-213 (2018).

76. Priebe, G. P. \& Goldberg, J. B. Vaccines for pseudomonas aeruginosa: a long and winding road. Expert Rev. Vaccines 13, 507-519 (2014).

77. Canaparo, $\mathrm{R}$ et al. Recent developments in antibacterial therapy: focus on stimuli-responsive drug-delivery systems and therapeutic nanoparticles. Molecules 24, 1991 (2019).

78. Kwon, E. J. et al. Porous silicon nanoparticle delivery of tandem peptide anti-infectives for the treatment of pseudomonas aeruginosa lung infections. Adv. Mater. 29, 1701527 (2017).

79. Kumar, M., Curtis, A. \& Hoskins, C. Application of nanoparticle technologies in the combat against anti-microbial resistance. Pharmaceutics. 10, 11 (2018).

80. Theuretzbacher, U. \& Paul, M. Developing a new antibiotic for extensively drug-resistant pathogens: the case of plazomicin. Clin. Microbiol. Inf. 24, 1231-1233 (2018)

81. Czaplewski, L. et al. Alternatives to antibiotics-a pipeline portfolio review. Lancet Infect. Dis. 16, 239-251 (2016)

\section{Acknowledgements}

U.T. received federal funds from the German Federal Ministry of Education and Research (BMBF) to support this Review. The funder had no role in study design, data collection, data analysis, data interpretation or writing of the article. K.O. is Principal Investigator and Executive Director of CARB-X funded by Notice of Award 5IDSEP160030-04-00 from the US Biomedical Advanced Research and Development Authority (Assistant Secretary for Preparedness and Response, Department of Health and Human Services), preclinical services from the US National Institute for Allergy and Infectious Diseases (National Institutes of Health, Department of Health and Human Services) and funding from the Wellcome Trust, the German Federal Ministry for Education and Research (BMBF), the Global AMR Innovation Fund from the UK Department of Health and Social Care and the Bill \& Melinda Gates Foundation. A.E. is a partner with
Novo Holdings A/S and Director of the REPAIR Impact Fund, which was commissioned by the Novo Nordisk Foundation. A.K. is the leader of the managing entity of the ENABLE consortium and has received support from the Innovative Medicines Initiative Joint Undertaking under grant agreement no. 115583, resources of which are composed of financial contributions from the European Union's seventh framework programme and European Federation of Pharmaceutical Industries and Associations companies' in-kind contribution. Those projects in ENABLE that volunteered information for this Review are gratefully acknowledged. The views herein do not necessarily represent the view of the ENABLE partners. The views expressed herein do not necessarily represent the views of CARB-X or any CARB-X funder, the REPAIR Impact Fund or ENABLE or any of its funders. The authors thank $L$. Marin for providing data from the Joint Programming Initiative on Antimicrobial Resistance.

\section{Author contributions}

A.E. provided the data from the REPAIR Impact Fund. K.O. provided the data from CARB-X. A.K. provided the data from ENABLE. U.T. provided the data from the Center for AntiInfective Agents (CEFAIA). All data was provided to U.T. for application of the inclusion criteria, descriptive results, analysis and discussion of the findings. U.T. wrote the first draft and K.O. provided the first comprehensive edit, which was then reviewed and edited by all authors.

\section{Competing interests}

The authors declare no competing interests.

\section{Publisher's note}

Springer Nature remains neutral with regard to jurisdictional claims in published maps and institutional affiliations.

\section{Supplementary information}

Supplementary information is available for this paper at https://doi.org/10.1038/s41579-019-0288-0.

\section{RELATED LINKS}

\section{ARB-X: https://carb-X}

.org/portfolio/gallery/

AB

Joint Programming Initiative on Antimicrobial Resistance:

https://www.jpiamr.eu/supportedprojects/

Pew Trusts: https://www.pewtrusts.org/en/research-and-

analysis/articles/2018/09/21/the-shared-platform-

for-antibiotic-research-and-knowledge

REPAIR Impact Fund: https://www.repair-impact-fund.com/ portfolio/ 